\title{
TEACHING EXPERIMENTS FOR ENGINEERING EDUCATION BASED ON CLOUD CAD SOFTWARE
}

\author{
Gaha, Raoudha; \\ Nicolet, Pierre-Marie; \\ Bricogne, Matthieu; \\ Eynard, Benoit
}

Roberval Laboratory - UMR CNRS 6253 -Sorbone Universités - Université de Technologie de Compiègne, Centre Pierre Guillaumat, BP 60319, Rue du docteur Schweitzer, F.60203 Compiègne Cedex, France

\begin{abstract}
Today we live a high increasing digitalization in industry 4.0. As part of the evolution of CAD solutions on the market, there is a particular interest in new generation software which are distributed as Software-as-a- Service (SaaS) such as Onshape, 3D Experience, Fusion 360, etc. In order to prepare engineering students for integrating such software within the Université de Technologie de Compiègne (UTC), a further practicing study is carried out in this paper. This study aims to identify, analyze, experiment, evaluate and compare the capacities of Cloud-based CAD solutions on the market and in scientific research work in order to define potential benefits for their implementation in mechanical engineering education. Therefore, we tested two use scenarios of the two Cloud-based software; Onshape and Fusion 360 on a case study. Then, we discussed the comparison results.
\end{abstract}

Keywords: Computer Aided Design (CAD), Education, Design engineering

\section{Contact:}

Gaha, Raoudha

Universite de Technologie de Compiegne

Ingénierie Mécanique

Tunisia

raoudha.gaha@utc.fr 


\section{INTRODUCTION}

Today, amid the significant growth of cloud-based technologies, advanced manufactures need engineers with a basic understanding of these technologies to stay competitive. As a result, traditional design methodologies are currently insufficient. Thus, teachers must adapt teaching and train competent graduates for the job market. These trends have major implications for engineering education; first, on universities (today no longer need to invest high-performance computing resources in the presence of affordable and easy-to-use cloud applications), second, on future engineers, who will include an industry 4.0 based on the Internet of Things (IoT). Today, the number of cloud-based software continues to grow. Hence, teachers must choose from the available systems and develop implementation strategies within the engineering training. Some platforms are hosted entirely in the cloud and can be used through a generic browser (e.g Onshape $\AA$ and Fusion $360 \AA)$. Others rely, mainly, on the Cloud for data storage but they require the download of a heavy client on a computer (3DExperience).

To study the possibility of integrating this type of software into the Mechanical Engineering course at University of Technology of Compiègne (UTC), an analysis based on the functionalities transmitted to the students, in the geometric modelling value unit (TN20 - (http://moodle.utc.fr/course/view.php?name= TN20)), throughout using classic software such as Catia and Creo, was made on CAD software in SAAS mode Fusion 360 and Onshape. This analysis allows, evaluating and comparing the possibilities offered by CAD software in SAAS mode and look at its implementation in an educational setting such as that of UTC. Thereby, we first conducted a literature review on the study of cloud-based solutions in universities training engineers. This state of the art allowed us to extract some criteria to be taken into consideration in our analysis as well as to know the advantages and disadvantages of software in SAAS mode and to compare them with our experiment on the two-cloud-based software CAD (Fusion 360 and Onshape), in the third section. A discussion of the results of the analysis is conducted in the fourth section, which is followed by a conclusion of the study presented in the fifth section.

\section{LITERATURE REVIEW}

\subsection{Related works}

Experimentation of cloud-based software is not new. Several universities have tested the implementation of these technologies in the field of engineering either within the framework of collaboration as Product Life Management systems (PLM) (Maranzana et al., 2012), (Fielding et al., 2014), (Bedolla et al., 2017), design (Eiden and Apostolov, 2017), (Junk and Spannbauer, 2018), or collaborative design (Barrie, J. 2016). In the development of products, there is no doubt that mechanical CAD software takes an important role in product design. Today, the complexity of products has clearly increased, then design engineers are facing numerous challenges such as the conflict in versions, costs in hardware and license when using desktop CAD software. Cloud-based emerging solutions, seem resolve these problems. However, the ability of cloud-based CAD software is still in question. That's why numerous researches are developed to evaluate and compare between desktop CAD systems and cloud-based ones. Junk and Kuen, (2016). Presented a review of open source and freeware CAD systems (e.g Autodesk FreeCAD, SketchUp and Onshape for Mechanical Construction, OpensCAD and RepoCAD for Programming and Blender for Creative design). They compared them from a scientific perspective based on two clusters of relevant criteria "ease of use" and "scope of functions" where a weighting factor is additionally implemented in the evaluation. This allows us to identify a first advantage, which is ease of use and leads us to use the criteria "Intuitive interface" and "software familiarization". Rassovytska and Striuk (2018). Realized a comparison between more than 30 various cloud services and mobile applications based on criteria defined by mechanical engineers' professional activity and the principles of their use in higher technical education. The most famous of them are A360, Fusion 360, GstarCAD, DWG FastView-CAD Viewer, CAD Pockets, 3D CAD Models Engineering, CAD Assistant, Onshape, GrabCAD, GnaCAD etc. The main criteria for selection are: functionality, availability, easy access from different devices, ability to integrate with other software, support for collaboration, this leads us to consider two additional comparison criteria: accessibility and collaboration. Le (2018) examined two prominent parametric modelling software: Solid-Works and Onshape based on SWOT analysis and AHP model in order to have a rational comparison. The author mentioned that, according to AHP tool, the comparison results presented that Onshape was most preferred with the final priority of 59\%, and SolidWorks as a runner 
up with its priority of $41 \%$ approximately, which further confirms the choice of our criterion "Software familiarisation". Junk and Spannbauer (2018) realized a technical comparison between Onshape and Fusion 360 based criteria related to daily use (e.g. userfriendliness and mobility), the evaluation of functionality (e.g. part design, virtual assembly and drawings) and functions that are rarely used or that run in the background (e.g.storage and getting started). Therefore, the design was taken into account as a comparison criterion, while considering the different functionalities to be acquired in TN20. They concluded that, Onshape offers the user a simple user surface, high mobility and flexibility in the extension of additional applications. Compared to Fusion 360 which has a large range of functions in a full package that contains many useful features, however it can have minimal extension. Among numerous CAD software today, and numerous comparisons between them, it hard to provide a performed evaluation. That's why, Galimova (2020) presented a summary table of the advantages and disadvantages, on the basis of which the author developed a method facilitating the choice of the optimal approach to CAD testing. Also, Wu et al. (2017) presented a technological guide for commercial customers to select the most appropriate application on the cloud or to use it during the whole product process development. This guide is generated from the state-of-the art that they realized on digital design and manufacturing software and services currently available on the cloud. Hence, they assessed what extent engineering design, engineering analysis, manufacturing, and production when performed based on the software through the cloud and then they extracted key capabilities and benefits of such software. This guide can be also practiced for educational issues (Yip-Hoi, 2020). $\mathrm{Wu}$ et al. (2015) identified the following common key characteristics of Cloud-based design manufacturing (scalability, agility, high performance and affordable computing, networked environments, ubiquitous access, self-service, big data, search engine, social media, real-time quoting, pay-per-use, resource pooling, virtualization, multi-tenancy, crowdsourcing, IaaS, PaaS, HaaS, and $\mathrm{SaaS})$. Then, they developed a requirements checklist for selecting the most appropriate CBDM (Cloud-Based Design and Manufacturing) system. For educational aim, onshape is experimented by students the University of Kaiserslautern, where they organized the development process by themselves using a full cloud CAD software. They deduced that Onshape is very powerful for collaborative work since it offers best data management functions like branching, versioning or merging of CAD models, hence they obtained complete results because all the data is already at the database (Eigner and al., 2017). Barrie (2016) presented Fusion 360 and Onshape as the two current and well know cloud-based CAD packages distributed as Software as-a- Service (SaaS) in the market. The author, points to the potential of such Software in an academic setting processed to a specific evaluation between both systems, by looking at the platforms, without expressing preference. At the end, as a provision perspective, he mentioned that cloud-based CAD could provide more flexible and universal access to CAD software across an institution.

The growing importance and interest to Cloud-based systems is highlighted in the researches cited above. In the next section we pointed on the advantages and limitations of using such software in educational field.

\subsection{Advantages and limitations of using cloud-based CAD software in education}

In the educational context, cloud-based CAD software system, which perform as a Software-as-aService (SaaS) have important benefits such as:

- Models and visualizations are supported by browsers enabling display on any device (Barrie, 2016)

- Wok file are replaced by compound documents, which are stored on the cloud as database entities

- Buying a costly node-locked annual license is not required.

- Versions and maintenance are updated automatically in the cloud

- Offering more flexibility and proliferation of use since students can access from any computer without specialist CAD hardware without conducting the class in a computer lab.

- $\quad$ Etc.

The list of advantages is not exhaustive, though, let's list some limitations found on the literature:

- Collaboration benefits are yet to be explored,

- Institutions have already their CAD platforms in place

- A poor Wi-Fi signal could disrupt the workflow in the cloud

- Etc. 
Fusion 360 and Onshape are distributed as a Software-as-a-Service (SaaS) and as mentioned on section 2 they served for comparison to explore cloud-based CAD benefits. In fact, Fusion 360 is a cloud-based 3D CAD, CAM, and CAE platform for product development, according to Barrie (2016) can offers industrial and mechanical design applications in the form of surfacing and solid modelling, operate on multiple platforms such as PC's, Macs and mobile devices and keep up to date with model and project changes in the cloud Fusion 360. Wu et al. (2017), mentioned that fusion 360 allows creating smooth and precise surfaces with T-Splines technology or with sketch curves, patches, and extrusions via a web browser. However, it does not facilitate global collaboration to unlike Onshape, which claims 'full-cloud' based CAD and collaboration. Indeed, its part-studio interface offers real time editing of features between multiple engineers, with clear edition in the feature tree (Barrie, 2016). enables multiple users to create new features on existing 3-D models or modify existing features that assists users in a flexible approach, such as Merge/Branch features (Le, 2018).

\section{EDUCATIONAL EXPERIMENTATION ONSHAPE AND FUSION 360}

In order to train students on CAD software in SAAS mode, the implementation of Onshape or Fusion 360 in TN20, with the objective of geometric modelling, is proposed at UTC, as part of a project semester carried out by a student in fifth year, having already done TN20 on Catia and Creo, under the supervision of two teacher-researchers, a Professor and a lecturer, who teach TN20. The student intern took over the geometric modelling of a taught case study, which is an automotive spring damper (Figure 1). This damper is used to perform a comparison based on two scenarios (modelling on Onshape and modelling on Fusion 360). The comparison studied the following criteria: the intuitiveness of the interface, accessibility, software familiarization, collaboration, design (volume, generative form and assembly) and conversion. These criteria come from the bibliographic study, on the one hand, and from TN20 objects, on the other hand.

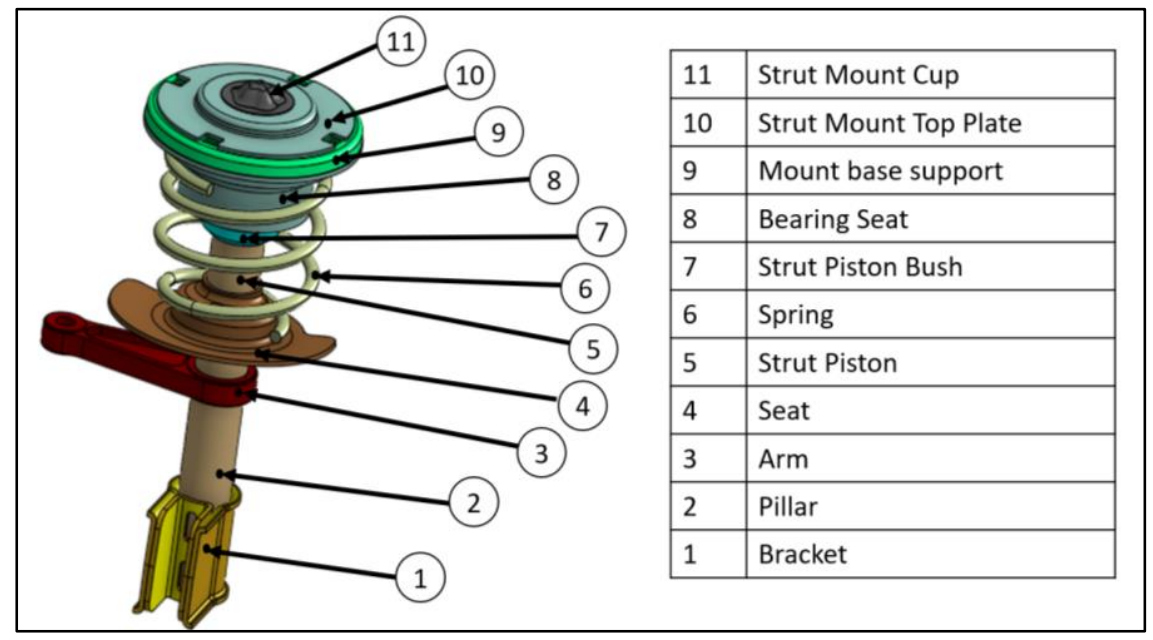

Figure 1 Product case study (Spring Damper)

\subsection{Intuitive interface}

The user interface in Onshape has similarities with Creo. In fact, features in the interface part studio and the tree structure are the same as on Creo and the feature search function is highlighted. Also, the system version is accessible without prior research and upgrading a part and its history are easily understandable. For Fusion 360, the novice user find that the interface is simpler than a classic software (such as Catia V5). Indeed, he is not overwhelmed by a multitude of functions on the screen, only the most used are displayed directly on the screen (sketch, extrusion, etc.). The surface ("Surface") and volume ("Solid") workshops make it easy to determine the type of design desired and thus avoid certain errors in the use of features. Also, it is noticed that there is the "Sheet metal" workshop but it has not been used here. However, the use becomes a little more complicated because many functions are "hidden" in the menus "create", "modify" or "assemble". Which can confuse a new CAD user. The tree is less accessible than OnShape's, but better ordered: it contains a few peculiarities that may confuse the user at first. Indeed, the tree is represented by functions (sketches, 
bodies or components) and the features for adding or modifying material are located at the bottom of the screen, which requires a little research during the first use. In addition, the list of these features is not very large and is rather difficult to read. However, to go back and modify a revolution for example, you have to go back to the bottom of this screen and click on the feature. Upgrading parts is easy because all you have to do is register the part, just like with any conventional CAD software.

\subsection{Accessibility}

In terms of accessibility, Onshape does not require installation on computer. It is $100 \%$ accessible on the web browser, and regardless the characteristics of the computer, the software will have the same fluidity on a computer with poorer characteristics than on a more powerful computer. However, Fusion 360 requires a light installation, but it is still CAD software designed on the principle of SAAS. Hence, a stable internet connection is required for Onshape, even though, while the light installation allows Fusion 360 to be used without an internet connection, although the user can, as well, use it online. This is convenient because even with an unstable connection, the software did not ask to refresh the page when a connection was lost. Also, from the accessibility point of view, Onshape is available as a mobile application, where 3D modelling can be realized in the same way as on a computer, but this poses ergonomic problems. Hence, it is mainly used for posting comments or previewing parts. Onshape is functional on many browsers such as Google Chrome, Mozilla Firefox, Safari (Mac OS only), Opera and Microsoft Edge. Regarding Fusion 360, the publisher recommends computer features that are widely affordable in the market: 3GB of storage, 4GB of RAM, graphics processor with at least $1 \mathrm{~GB}$ of dedicated RAM. A lighter version of Fusion 360 is available in a browser but has not been tested here.

\subsection{Software familiarization}

The software is taken up fairly quickly, especially in terms of the basic functions of extrusion, revolution, scanning, Boolean, etc. where the system is always the same. Indeed, features, Surfaces and parts can be manipulated at the same interface. Thus, some subtleties are to be grasped (assemblies are not based on plans but on points and connectors) because all functions are based on sketches. Also, Fusion 360 gets started quickly when creating parts for the first time. However, some subtleties like the tree structure and the presence of features at the bottom of the screen can be unsettling. In addition, the software uses concepts that are specific to it such as "bodies" and "components". Moreover, in Fusion 360, the user has the possibility to create assemblies of parts from parts created in the same "design" (workspace), the parts are all found in the same space and are not distributed in a directory. Thus, the "body" function will create a body distinct from the first bodies created. The same applies to components, but a component allows movements to the part, and not the body.

\subsection{Collaboration}

From the side of Onshape, the collaborative system is very intuitive. It strongly resembles collaborative drives like Google drive; the share of a part or a document containing parts or data, can be done individually or with a team, while managing their access rights. Also, users without an Onshape subscription can see a part if a link to it part is shared with them. In addition, a project system exists, in which roles are distributed. A test collaboration was then carried out where two students were connected at the same time on the Workspace of a designed part. When the first student creates a sketch (without validating it), the second connected student have the rights for modifications, hence, he can access the sketch and modify the dimensions or even add an extrusion. Thus, the part upgrading does not, therefore, condition added features access. From Fusion 360 side, the collaborative part is quite close to a PLM system. It integrates two concepts: hubs and projects. The projects are integrated into hubs. Each hub has a team in which users have different roles (team administrator, team member, project contributor). Each role incorporates certain rights. Likewise, each project includes three roles, which authorize some actions (administrator, editor, viewer). These roles are issued based on the user's role in the hub. In terms, of real-time collaboration, the software shows the other users working on the project or part. Unlike OnShape, it is not enough to create a sketch for the other user to see it, therefore the part has to be saved, so that the other logged in user can see what has been done. 


\subsection{Design (Volume, Generative shape, and Assembly)}

\subsubsection{Designing with Onshape}

The design is done in a "Workspace". The workspace can contain several parts in the same space. This part is imaged by designing an automotive spring damper using the skeleton method. Onshape is based on three essential principles: the sketches (which will feed all the features even the holes), the type of the developed object (part-part - surface or feature) and the Boolean (new, add, remove, intersect).

To create a volume (figure 2), the user has to go through the creation of a sketch first. It is not possible to create a sketch in the create extrusion menu. For pattern, revolutions and symmetries it is also necessary to specify whether the objects are features. It is, moreover, noticed that the axis system does not exist on Onshape. In fact, if the user wants to take as reference an axis passing through the center of a cylinder, he has to create it first. In Onshape, designing a spring, requires creating a sketch to model the section, and a sketch to model the diameter of the neutral spring fiber. Hence, this software allows more accessible solid modelling to $\mathrm{CAD}$ novice users than Creo, thanks to the functions that facilitate its use.
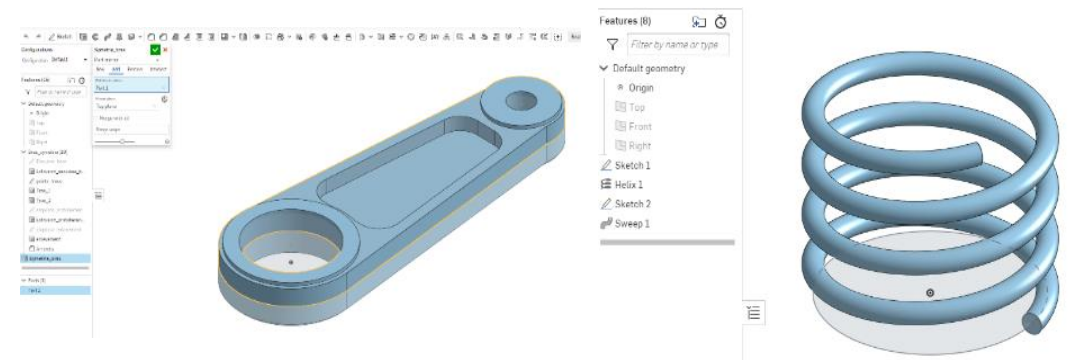

Figure 2 Volume creation on Onshape

To model the part "Strut Mount Cup" (figure1), two profiles are created (top and bottom) of the part on sketches in two different planes, and connect them using the "loft" function. It could incorporate a guide curve as well, but this function is not used in this example. Once again, the Boolean functions and the notion of shape/volume noticed can be noticed. Adding volume is done in the same way as with conventional software, choosing the area to thicken and the direction (inside, outside, both directions). Brackets were then added in volume via a sketch and by circular repeat.

The assembly performed in this test is based on the positioning skeleton. In Onshape, created blueprints do not appear while in the assembly shop (and cannot be viewed) because assembly on Onshape goes through points called "mate connector". These points are either created in a sketch or on a solid. For example, a revolved volume will have a mate connector in the center of the end of the part. In the example, the plate is attached to the tree by the point in the center of the circular extrusion (which was not created) to the point of the skeleton (which was created). Thus, the skeleton contains points instead of planes. In this example, the plate has been fixed ("fastened") to the shaft but a revolution around the shaft can be made. This system is therefore different from that of Creo, which works by mechanical links (coincidence, coplanar contact or distance). It is possible in a connection at one point to completely fix a part, this can pose a problem when we want to add the connections between two parts. The system can also be disconcerting because the part can be oriented in a way that the user does not want, it is then necessary to play on the revolution of the part according to the different axes of the global coordinate system. The tree structure of the assembly workshop is very simple: it only lists the parts added to the assembly and the connections made between the parts. When user inserts an object into an Onshape assembly, he can insert a part on the part of the studio. If a studio part contains several rooms, he can integrate as many rooms as he wants from that studio. However, if the studio part contains only sketches (as in the case of the skeleton method), the software will only include sketches. If there are 2 sketches in the studio part, the assembly will contain these two sketches, and each sketch will be brought to the same level as a part. It can also be noted that the software considers the version updates of the parts (but only if a new version of the part has been created). If a part is modified but a new version of the part is not created, the software will not send a notification about it; the user, then, must force the update by right clicking on the part in the assembly and "update link document". 


\subsubsection{Designing with Fusion 360}

The design is done in a workshop called "design". It can contain several parts in the same space (see the notions of bodies and components). The part, automotive spring damper (figure1), is modelled based on the skeleton method. The Fusion360 software is mainly based on sketches such as with Onshape.

Solid Fusion 360 works as the same way of Onshape (and regular CAD software). Thus, the sketches are the basis of features, and the features are the same as those on classic software. As on Onshape, the notions of bodies and components must be taken into consideration when using features. Although they can be annoying at first, these functions are interesting because they allow to create an assembly of parts themselves designed in the same workspace ("design"). The advantage is that all the parts are in same workspace and that the user does not have to navigate through the files of the Hub to insert created parts. The modelling of a spring was very easy thanks to the "Spire" feature (figure3), which in fact does not require any sketch and which includes all the input data (section, pitch, diameter of neutral fiber, etc.). Another interesting function, it is possible to create several fillets or chamfers in a single function, even if the values are not the same. This is not the case on Onshape.

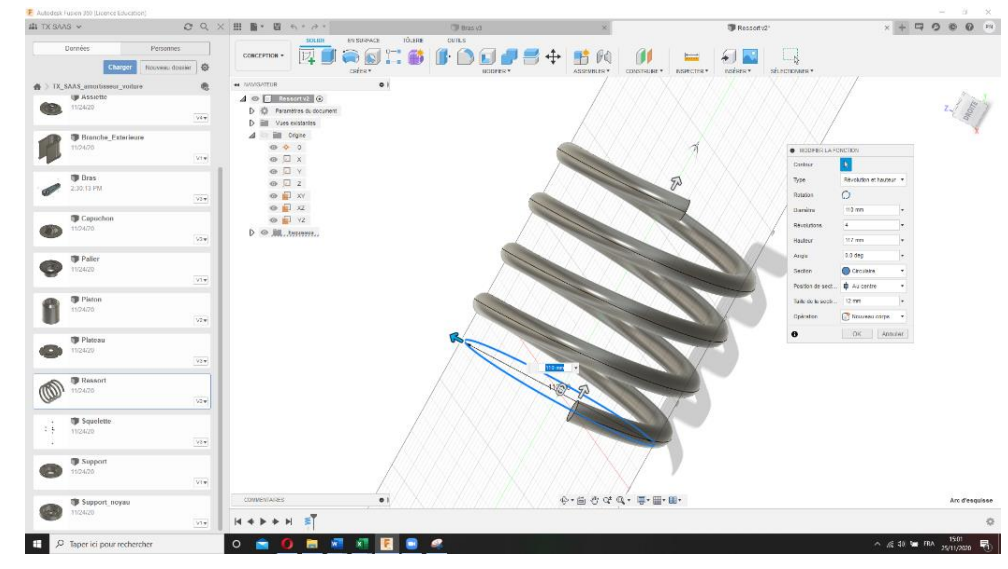

Figure 3 Spring design on Fusion 360

The shape design on Fusion 360 is realized on the same way on Onshape (and therefore other classic CAD software). The modelling of the part "strut mount cup" was the same for both software. The only difference seen between Onshape and Fusion360 was the circular pattern. Indeed, the parameter indications are clearer on Fusion360 than on Onshape. The assembly, made in this example, is still based on the skeleton method. What is special about Fusion360 is that there is no "assembly" workshop. All the design functions (volume or surface) are present in the same workspace as the assembly. In Fusion360, created drawings do not appear while in the assembly and can be used as a reference to assemble, unlike Onshape. It is, still, noted some common points between the two software, because Fusion 360 also offers positioning via points, with exactly the same system, where it is possible to fix one part to another with a single point. As on Onshape, these points are either created in a sketch or implied on a volume. In the example, the part "seat" (figure 1), is attached to the tree by the point in the center of the circular extrusion (which was not created) to the point of the skeleton (which was created). Thus, the skeleton contains points and planes that can each serve as a reference. On Fusion360, assembly is easier because it is more intuitive.

In Fusion 360, the system can, also, be disconcerting because the part can orient itself in a way that the user does not want, it is then necessary to play on the revolution of the part according to the different axes of the global coordinate system. Fusion360's tree structure is more complete than Onshape's. Every sketch, created in a part, can be seen, directly in the tree. However, to modify a part, we still have to modify it in its assembly context, like on Onshape. When we insert an object in Fusion360, we insert all the content of the design. If there are multiple bodies in the design to be inserted, the software integrates all of these bodies, unwanted ones have to be removed. We can also note that the software considers the updates of the parts only if they are recorded, as in Onshape.

\subsection{Conversion}

Exporting shapes, in Onshape, can be done into Parasolid, ACIS, STEP, IGES, SOLIDWORKS, and Rhino.The parts can be exported in DWG and DXF and drawings can be exported to DWG and DXF. 
Plans and sketches can be exported into Parasolid, ACIS, STEP, IGES, SOLIDWORKS, Collada, Rhino, and GLTF. For importing an assembly several solutions exist. Thus, it is possible to import a complete assembly and to request to separate the parts in different Part studio. It is also possible to import everything into a single Part studio, or put the assembly of the whole in an assembly shop and the other parts in parts studios. In this example, the whole assembly and sub-assemblies are in assembly shops and the other parts are in studio shares.

From his side, Fusion 360 can export in. f3d (which is the format specific to Fusion360), .ipt, .dwg, .dxf, fbx, .igs / .iges, .obj, .sat, .skp, .smt, .step, .stl. for parts and assemblies and in .pdf, .dwg, .dxf, .csv for definition drawings. When importing an assembly into Fusion 360, the software integrates the assembly of the assembly with the same subassemblies created, as in OnShape. And like in OnShape, the software only integrates the body of the part.

\section{EXPERIMENTATION RESULTS AND DISCUSSION}

In order to have concrete results and to demonstrate what is different between the two platforms, we have defined the criteria mentioned above. The marks, shown in Table 1 for the comparison of the functionalities of the two software, were given keeping in view the fact that the software would be used by students entering the mechanical engineering course, not necessarily having used CAD software before. Thus, the intuitiveness of the interface will raise the question of what a CAD novice sees when he opens the platform: is there too much information on the screen? Is the platform affordable and understandable at first glance? From where the criterion "intuitiveness of the interface" was determined as a moderately important criterion since it is not this criterion that will determine if the software is complex or not, once the user knows where to go. search for the desired feature, this criterion no longer appears. For this criterion, a significance coefficient of $6 / 10$ has been assigned. For this criterion Onshape received a score of 9/10 and Fusion 360 received a score of 7/10.

Accessibility depends on the conditions of use of the software: does the student have to install (heavy or light)? Does he need a computer with certain specifications? Does it need to have a highperformance internet connection? This criterion is so important to ensure smooth training for students, hence, it is very important for the student to be able to access the software from his personal computer, without encountering any problem. As a result, it was assigned a coefficient of $9 / 10$. For this criterion, Onshape is rated 6/10 and Fusion 360 is rated 9/10.

The "design" criterion links the software directly to its main use: design. We are talking here about all the features related to design, and modelling workshops. The underlying questions relate to the possibilities offered by these software via their functionalities: what features are present or absent, and does this pose a problem in the design of the system? This criterion is the average of the volume, surface designs, and of the assembly, which are considered primary objectives of TN20 formation. As a result, a 10/10 coefficient was assigned for this criterion, the main objective of this study. The rating given to Onshape, after experimentation, is 7.7 / 10 while Fusion 360 received a rating of 8.3 /10.

The criteria "software familiarisation" takes into account the time it takes for a new user to adapt to the software: does the software operate similarly on the features, and if so, does this make it easier to use? For example, we can note that on Onshape, the interface will often be the same on the different features: surface or volume, then Boolean operations, then we select the entities on which we work. For this criterion a coefficient of 7/10 was assigned, for which Onshape received a score of 7/10 and Fusion 360 was rated 6/10.

The criterion of collaboration is important since it is a very interesting additional functionality compared to traditional CAD software. In addition, the CAD learning subject is done in pairs at the University of Technology of Compiègne. This led us to determine that collaboration is an important criterion, especially as the cloud and the collaborative system are becoming more and more important in companies today. This is why it was assigned a coefficient of $8 / 10$, on which Onshape and Fusion 360 got the same rating of $8 / 10$.

The conversion refers to the possible export and import formats. These formats allow interoperability between different software. In addition, in the context of an evolving digitization in an industry 4.0, it seems to be a fairly important criterion as well as collaboration. Hence, this criterion was defined at the same level as the collaboration and it was assigned a coefficient of $8 / 10$, on which the two software tested do not seem to be mature enough receiving a similar score of 6/10. 
The additional functionalities are just a plus. Hence, they were assigned a coefficient of $3 / 10$. For this criterion Onshape received a score of 7/10 while Fusion 360 received a score of 8/10.

We can notice, from Table 1, that the scores are relatively close and that each software has its peculiarities. Thus, Fusion 360 has more features and is therefore easier to use for design, but is less intuitive than Onshape, which can lose a CAD novice.

Table 1Technical comparison between Onshape and Fusion 360

\begin{tabular}{|l|l|l|l|}
\hline \multicolumn{1}{|c|}{ Software } & Onshape & Fusion 360 & Coefficient (/10) \\
\hline Criteria & 9 & 7 & 6 \\
\hline Antuitive Interface & 6 & 9 & 9 \\
\hline $\begin{array}{l}\text { Design Shape, Volume, Assembly } \\
\text { (Skeleton method) }\end{array}$ & 7,7 & 8,3 & 10 \\
\hline Software familiarization & 7 & 6 & 7 \\
\hline Collaboration & 8 & 8 & 8 \\
\hline Conversion & 6 & 6 & 8 \\
\hline Additional functionalities & 7 & 8 & 3 \\
\hline Average & 7,2 & 7,53 & \\
\hline
\end{tabular}

\section{CONCLUSION}

As part of the implementation of a geometric modeling software for mechanical engineering training at UTC and more precisely for the TN20 teaching unit, we launched the experimental project for two software Onshape and Fusion 360 , to study their capacities to be used by students generally working in pairs or even in trinomials. This experiment focused on the evaluation of the two software programs on certain criteria (intuitiveness, handling, accessibility, collaboration, design and conversion) which are, either from the literature review that we developed in the first section, are derived from the objectives of TN20. This study showed that Onshape has a more attractive side than Fusion 360, especially since its philosophy is the same for all features, so an error is quickly spotted. Fusion 360 is more interesting than Onshape, at the assembly level, where the Onshape system is a bit difficult to grasp. The choice of assembly by skeleton method was not good, the software already making it possible to collaborate effectively via the comments and annotations sections, as well as by the functionalities already present. In addition, Fusion 360 can be used offline, which is a significant advantage, especially in the case of unstable internet connection, where disconnections are very frequent on Onshape.

Like all CAD software in SAAS mode, Onshape and Fusion 360, are not subject to heavy maintenance. Indeed, software updates in SAAS mode are done directly from the software publisher's servers, so the customer does not have to manage any software upgrades. During an update, the customer will connect to his software and will then have direct access to the latest version of the software. Fusion 360 requires a light installation on the local computer (this is what allows the software to be used offline), which can potentially require a little management of this installation on a company's computers. In addition, updates are made on the publisher's servers, the data is managed remotely (but can be imported if you want to work offline). Onshape maintenance is next to zero, as it is fully accessible through a browser.

Both software have various advantages, starting with storage. Indeed, the cloud allows users to not have to manage their data locally. Investing in various expensive equipment such as servers. The amount of storage will depend on an agreement between a software publisher and the institution. This notion of storage still has some drawbacks, so a company will no longer have control over its data because it will be present on the publisher's servers.

At the end of this study and based on the comparison made, we are moving towards the use of Fusion 360 having an average slightly higher than that obtained by Onshape, based moreover on the condition of connection stability, Fusion 360 will be able to ensure the progress of a TN20 session without worry. 


\section{REFERENCES}

Barrie, J., 2016. “Applications for cloud-based CAD in design education and collaboration”. In DS 83: Proceedings of the 18th International Conference on Engineering and Product Design Education (E\&PDE16), Design Education: collaboration and Cross-Disciplinarity, Aalborg, Denmark, 8th-9th September 2016, pp. 178-183.

Bedolla, J. S., D’antonio, G., Segonds, F., \& Chiabert, P. (2017, July). "PLM in Engineering Education: A Pilot Study for Insights on Actual and Future Trends". In IFIP International Conference on Product Lifecycle Management pp. 277-284. Springer, Cham. https://doi.org/10.1007/978-3-319-72905-3_25

Eiden, A., \& Apostolov, H. (2017). Crowd Engineering-Bringing Full Cloud CAD into the Lab. "In DS 88: Proceedings of the 19th International Conference on Engineering and Product Design Education (E\&PDE17), Building Community: Design Education for a Sustainable Future", Oslo, Norway, 7 \& 8 September 2017 (pp. 170-175). ISBN: 978-1-904670-84-1

Fielding, E. A., McCardle, J. R., Eynard, B., Hartman, N., \& Fraser, A. (2014). "Product lifecycle management in design and engineering education: International perspectives". Concurrent Engineering, 22(2), pp123134. https://doi.org/10.1177/1063293X13520316

Galimova, E. Y. (2020, September). "Discussion of cloud CAD software testing approach". In IOP Conference Series: Materials Science and Engineering Vol. 919, No. 5, p. 052034. IOP Publishing.

Junk, S., \& Kuen, C. (2016). "Review of open source and freeware CAD systems for use with 3D-printing". Procedia CIRP, vol. 50, pp430-435. https://doi.org/10.1016/j.procir.2016.04.174

Junk, S., \& Spannbauer, D. (2018, April). "Use of cloud-based computer aided design software in design education". 17th International Conference on Information Technology Based Higher Education and Training (ITHET) (pp. 1-6) IEEE. http://doi.org/10.1109/ITHET.2018.8424769.

Le, N. (2018). "Product Design with Cloud Based and Desktop CAD software: A comparison between SolidWorks and Onshape”. Degree Thesis, Plastics Technology, Arcada University,Finland

Maranzana, N., Segonds, F., Lesage, F., \& Nelson, J. (2012, July). "Collaborative design tools: a comparison between free software and PLM solutions in engineering education". In IFIP International Conference on Product Lifecycle Management pp. 547-558. Springer, Berlin, Heidelberg. https://doi.org/10.1007/978-3642-35758-9_49

Rassovytska, M., \& Striuk, A. (2018). Mechanical engineers training in using cloud and mobile services in professional activity. arXiv preprint arXiv:1807.00313. Vol. 1844 (2017) pp348-359, arXiv:1807.00313

Wu, D., Rosen, D. W., Wang, L., \& Schaefer, D. (2015). "Cloud-based design and manufacturing: A new paradigm in digital manufacturing and design innovation”. Computer-Aided Design, Vol.59, pp1-14. https://doi.org/10.1016/j.cad.2014.07.006

Wu, D., Terpenny, J., \& Schaefer, D. (2017). "Digital design and manufacturing on the cloud: A review of software and services-RETRACTED”. Artificial Intelligence for Engineering Design, Analysis and Manufacturing, 31(1). Vol 31, 104-118. https://https://dx.doi.org/10.1017/S0890060416000305

Yip-Hoi, D. M. “Cloud Based Computer-Aided Engineering Education: Finding the Silver Lining”, ASEE'S virtual Conference, 22-26 June 2020 